\title{
Subgaleal Hematoma
}

\author{
Sascha Meyer • Ludwig Gortner • Hashim Abdul-Khaliq • \\ Panagiotis Papanagiotou
}

Received: 12 April 2013 / Accepted: 23 May 2013 / Published online: 4 July 2013

(C) Dr. K C Chaudhuri Foundation 2013

A 14-y-old boy presented to authors' emergency department (ED) with a fluctuant boggy mass over the scalp after minor traumatic brain injury. MRI studies demonstrated progressive subgaleal hematoma while no osseous lesions were seen on cranial CT scans (Figs. 1a and b). To rule out a clotting disorder in the patient both routine tests of blood coagulation, including the prothrombin time (PT), activated partial thromboplastin time (aPTT), and thrombin time (TT), fibrinogen and d-dimer concentrations, template bleeding time (Ivy method), full blood count to exclude thrombocytopenia as well as a detailed analysis of individual clotting factors (all came back as normal) were performed. Also, both the patient's past medical history and the family history were unremarkable with regard to an underlying coagulopathy.
The subgaleal hematoma was surgically removed with an uneventful post-operative course.

Subgaleal hematoma is caused by rupture of emissary veins and is typically seen in neonates after vacuum delivery $[1,2]$. It is a located between the periosteum and the scalp galea aponeurotica with the subgaleal space extending from the orbital ridges to the nuchal ridge with lateral confinement to the temporal fascia. Furthermore, a subgaleal hematoma is characterized by crossing of the cranial sutures [1,2]. In older children-as in index patient-it may be seen after minor head trauma or may be of nontraumatic origin. The hematoma often resolves spontaneously or with conservative treatment using a compression bandage (usually within a few weeks) [3]. In case of failure of conservative treatment, aspiration or surgery may be mandatory [1-3].
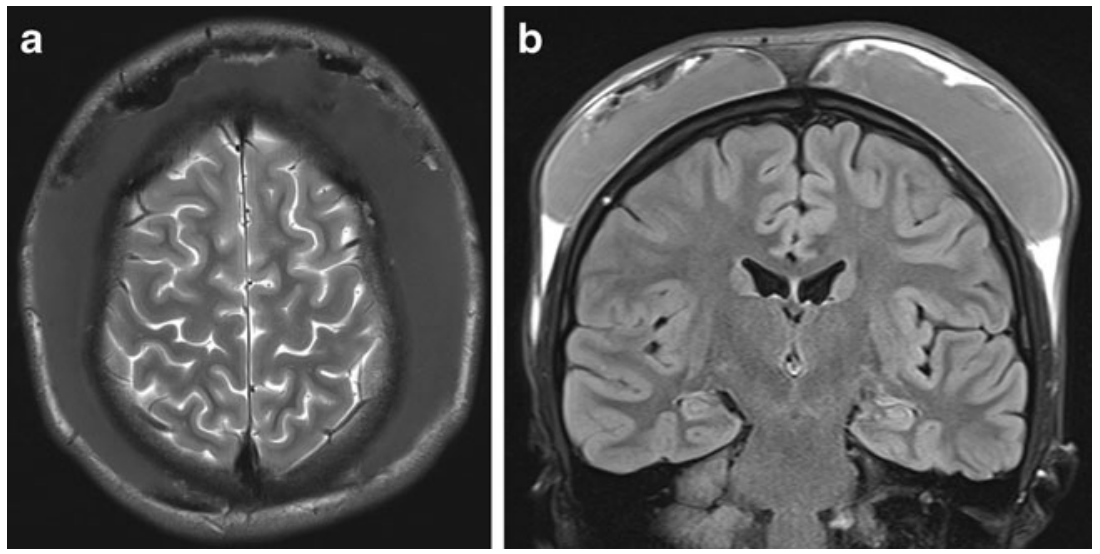

Fig. 1 a ( $\mathrm{T}_{2}$-weighed) and b (FLAIR): Cerebral MRI (axial and coronal view) demonstrating large subgaleal hematoma prior to surgery

S. Meyer $(\bowtie) \cdot$ L. Gortner

Department of Pediatrics and Neonatology,

University Children's Hospital of Saarland, Building 9,

66421 Homburg, Germany

e-mail: sascha.meyer@uks.eu

\section{References}

\section{H. Abdul-Khaliq}

Department of Pediatric Cardiology,

University Children's Hospital of Saarland, Homburg, Germany

\section{P. Papanagiotou}

Department of Neuroradiology,

University Hospital of Saarland, Homburg, Germany
1. Koizumi H, Suzuki S, Utsuki S, Nakahara K, Niki J, Mabuchi I, et al. A case of non-traumatic subgaleal hematoma effectively treated with endovascular surgery. Interv Neuroradiol. 2010;16:317-21.

2. Shehu BB. Massive chronic subgaleal haematoma. Br J Neurosurg. 2002;16:396-7.

3. Kichari JR, Gielkens H. Massive traumatic subgaleal haematoma. Emerg Med J. 2013;30:344. 